\title{
Материаловедение. Нанотехнологии
}

УДК 661.66-022.53

DOI: 10.17277/vestnik.2016.02.pp.323-333

\section{ОКИСЛЕНИЕ МНОГОСЛОЙНЫХ УГЛЕРОДНЫХ НАНОТРУБОК В ПАРАХ ПЕРЕКИСИ ВОДОРОДА: ЗАКОНОМЕРНОСТИ И ЭФФЕКТЫ}

\author{
Т. П. Дьячкова ${ }^{1}$, Ю. А. Хан ${ }^{1}$, Н. В. Орлова ${ }^{2}$, С. В. Кондрашов ${ }^{3}$ \\ Кафедры: «Техника и технологии производства нанопродуктов» (1); \\ «Технологические проиессы, аппараты и техносферная безопасность» (2), \\ ФГБОУ ВО «ТГТУ»; ФГУП «Всероссийский научно-исследовательский институт \\ авиационных материалов», г. Москва (3); dyachkova_tp@mail.ru
}

Ключевые слова: газофазный процесс; композиционные материалы; окисление; степень дефектности; углеродные нанотрубки; функционализация.

Аннотация: Представлены закономерности окисления многослойных углеродных нанотрубок в парах перекиси водорода. Исходные и обработанные углеродные нанотрубоки исследованы методами электронной микроскопии, ИКи РФЭ-спектроскории, спектроскопии комбинационного рассеяния, ренгеноструктурного анализа, сорбтометрии и термогравиметрии. Определено влияние температурных условий, продолжительности процесса и наличия примесей металлоксидного катализатора на качественный и количественный составы поверхностных функциональных групп, формирующихся при окислении углеродных нанотрубок в парах перекиси водорода. Проведена оценка влияния данного способа окисления на структуру графеновых слоев углеродных нанотрубок. Показано, что полимерные композиты на основе функционализированных углеродных нанотрубок обладают улучшенными радиоэкранирующими и электропроводящими свойствами. Предлагаемый способ обработки углеродных нанотрубок является экономически выгодным, экологически чистым и может быть легко масштабирован.

\section{Введение}

Углеродные нанотрубки (УНТ) - перспективные наполнители полимерных конструкционных материалов, улучшающие ряд механических и электрофизических характеристик $[1,2]$. Однако при введении в матрицы часто наблюдаются явления агломерации УНТ, значительно понижающие полезный эффект от их применения [3]. Изменение химического состава поверхностных слоев нанотрубок посредством функционализации способствует их более равномерному диспергированию [4], и, как следствие, значительному снижению требуемых расходных норм.

Наиболее простой и доступный методом обработки УНТ, повышающий их сродство к полярным полимерным матрицам - окисление в различных системах: концентрированной азотной кислоте и смесях на ее основе; перманганате 
калия; смеси перекиси водорода с аммиаком и т.п. Однако в ряде случаев, когда требуется сохранить морфологические характеристики исходных УНТ и включения металлоксидного катализатора в них, целесообразно применять методы газофазного окисления в горячем воздухе [5], парах азотной кислоты [6], озоне [7]. Первый из предлагаемых вариантов реализуется при достаточно высоких температурах - 300.. $400{ }^{\circ} \mathrm{C}$, второй и третий - требуют использования достаточно агрессивных и токсичных реагентов. В связи с этим актуальным является поиск эффективных газофазных окисляющих реагентов, у которых отсутствуют указанные недостатки.

Данным требованиям удовлетворяют пары перекиси водорода, которые, как показано в источниках $[8,9]$, применяются для окисления различных органических соединений при температуре $100 \ldots 160^{\circ} \mathrm{C}$. Включения частиц переходных металлов, являющихся компонентом металлоксидных катализаторов CVD-синтеза многослойных УНТ, катализируют разложение $\mathrm{H}_{2} \mathrm{O}_{2}$ на свободные радикалы, которые могут активно участвовать в окислении поверхности УНТ $[10,11]$.

\section{Методика эксперимента}

В работе использованы многослойные УНТ марки Таунит-М (производства ООО «НаноТехЦентр», г. Тамбов), диаметром 8..15 нм, длиной около 2 мкм, с удельной поверхностью - 300...320 м²/г, синтезированные CVD-методом [12] на катализаторе $\mathrm{Co} / \mathrm{Mo} / \mathrm{Mg} / \mathrm{Al}$. В ряде экспериментов использованы предварительно очищенные от примесей металлоксидного катализатора УНТ. Очистка осуществлялась обработкой в концентрированной $\mathrm{HCl}$ с последующей отмывкой дистиллированной водой и высушиванием полученной пасты УНТ в вакуумном шкафу при $60^{\circ} \mathrm{C}$.

Газофазную функционализацию УНТ осуществляли в парах 37\%-го водного раствора перекиси водорода (квалификации «ч.») при $120 \ldots 180{ }^{\circ} \mathrm{C}$ в течение 0,5...30 ч. Использована лабораторная установка, характеристики которой представлены в [13].

Первоначальная идентификация формирующихся при окислении УНТ поверхностных функциональных групп осуществлялась методом ИК-спектроскопии на приборе Infraluum FT-801 в волновом диапазоне $400 \ldots 550 \mathrm{~cm}^{-1}$. Для детального качественного анализа поверхностных образований применялся метод рентгеновской фотоэлектронной (РФЭ) спектроскопии с использованием оборудования Kratos Axis Ultra DLD (Великобритания).

Структура поверхностных графеновых слоев оценивалась посредством рентгеноструктурного анализа на рентгеновском дифрактометре «Дифрей-401». Степень дефектности УНТ оценивалась по соотношению пиков $D\left(\sim 1300 \mathrm{~cm}^{-1}\right)$ и $G\left(\sim 1500 \mathrm{~cm}^{-1}\right)$, регистрируемых на спектрах комбинационного рассеяния (КР) с помощью прибора DXR Raman Microscope (Thermo Scientific) при длине волны возбуждающего лазера 532 нм.

Термогравиметрический (TГ) анализ и дифференциальная сканирующая колориметрия (ДСК) исходных и функционализированных УНТ осуществлялся в атмосфере воздуха на оборудовании STA 449 F3 Jupiter (Netzsch, Германия) при скорости нагрева $10^{\circ} \mathrm{C} /$ мин. Удельная поверхность материалов определялась по адсорбции азота при 77 К многоточечным методом БЭТ на приборе Nova 1200e (Quantachrome, США).

Исследования структуры образцов выполнены с помощью сканирующей и просвечивающей электронной микроскопии (СЭМ и ПЭМ). Измерения относительной комплексной диэлектрической проницаемости нанокомпозитов на основе исходных и функционализированных УНТ в диапазоне СВЧ проводились на изме- 
рительном стенде, схема которого представлена на рис. 1; измерения поверхностного сопротивления нанокомпозитов - в соответствии с ГОСТ Р 50499-93 (МЭК 93-80).

\section{Результаты и обсуждение}

Предлагаемая окислительная система становится эффективной по отношению к УНТ при температуре выше $140{ }^{\circ} \mathrm{C}$. После обработки при данных условиях на ИКспектрах УНТ (рис. 2) присутствуют усиливающиеся по мере увеличения продолжительности процесса поло-

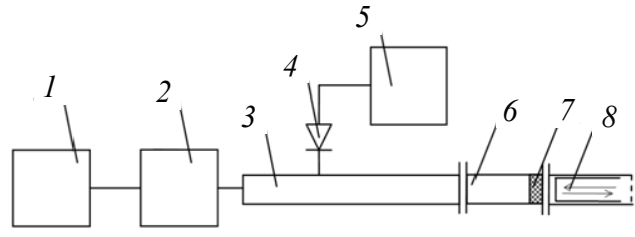

Рис. 1. Функциональная схема измерительного стенда для определения диэлектрической проницаемости нанокомпозитов:

1 - генератор СВЧ; 2 - аттенюатор;

3 - измерительная волноводная линия;

4 - перемещающийся зонд с детектором;

5 - усилитель низких частот с индикатором;

6 - измерительная ячейка; 7 - испытуемый образец; 8 - волноводная секция с поршнем

сы, подтверждающие наличие групп -ОН (3450 cм $\left.{ }^{-1}\right)$ и $-\mathrm{COOH}\left(1710 \mathrm{~cm}^{-1}\right)$. Степень функционализации окисленных УНТ довольно низкая, что определяется по характеру ТГ-кривых (рис. 3). Различия в термической стабильности исходных и функционализированных УНТ минимальны.

По данным ДСК-анализа термоокислительная деструкция исходных УНТ характеризуется более высоким тепловым эффектом, что связано с наличием в исходном материале включений аморфной фазы, которая должна иметь более высокие значения энтальпии сгорания, чем УНТ, по аналогии с данными величинами для сажи и графита [14].

Содержание химических элементов в образцах, рассчитанное по РФЭспектрам, приведены в табл. 1. Концентрация кислорода в обработанных парами перекиси водорода материалах незначительная, но она более высока в случае предварительно очищенных от катализатора УНТ. Наличие следовых количеств элементарного хлора в последнем случае обусловлено использованием соляной кислоты для удаления примесей. На основании полученных результатов можно судить о том, что частицы переходных металлов способствуют разложению $\mathrm{H}_{2} \mathrm{O}_{2}$, снижая при этом ее окисляющее действие.

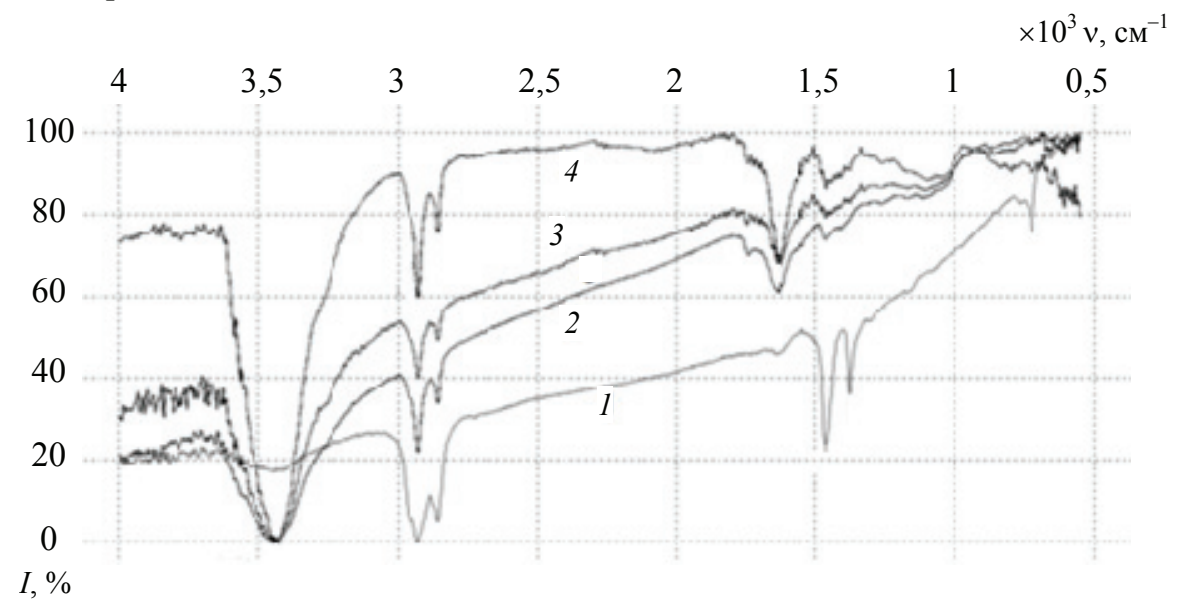

Рис. 2. ИК-спектры исходных (1) и окисленных в парах перекиси водорода при $140{ }^{\circ} \mathrm{C}(2-4)$ УНТ Таунит-М.

Время окисления, ч: $2-2 \ldots .5 ; 3-3 \ldots 15 ; 4-4 \ldots 20$ ( $v-$ волновое число) 


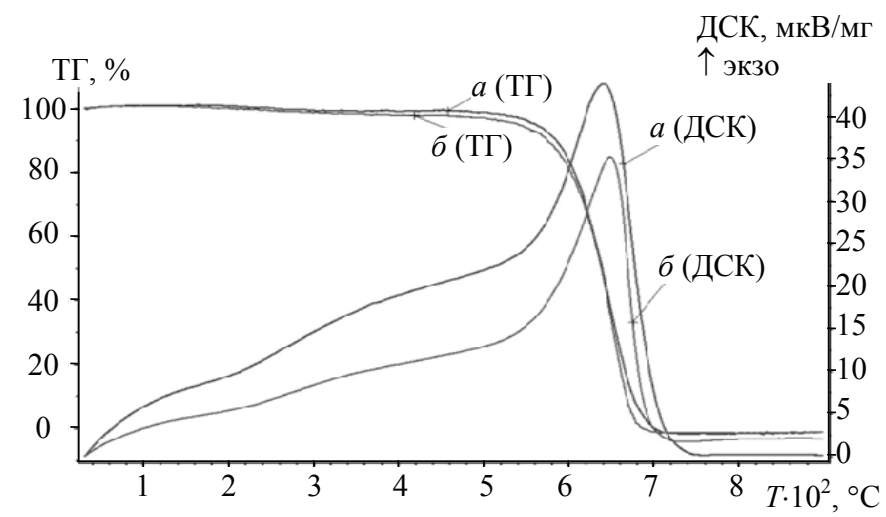

Рис. 3. ТГ- и ДСК-кривые исходных (a) и окисленных в течение 5 ч в парах перекиси водорода при $140{ }^{\circ} \mathrm{C}($ () $У$ УН Таунит-М

Таблица 1

Содержание элементов и доли состояний атомов углерода в образцах исходных и окисленных в парах перекиси водорода УНТ Таунит-М по данным РФЭ-спектроскопии

\begin{tabular}{l|c|c|c|c|c|c|c}
\hline \multirow{2}{*}{ Образец } & \multicolumn{3}{|c|}{ Содержание элементов, \% ат. } & \multicolumn{5}{|c}{ Доля состоянй, \% } \\
\cline { 2 - 8 } & $\mathrm{C}$ & $\mathrm{O}$ & $\mathrm{Cl}$ & $\begin{array}{c}\mathrm{C}-\mathrm{C} \\
\left(\mathrm{sp}^{2}\right)\end{array}$ & $\begin{array}{c}\mathrm{C}-\mathrm{C} \\
\left(\mathrm{sp}^{3}\right)\end{array}$ & $\mathrm{C}-\mathrm{O}$ & $\mathrm{O}=\mathrm{C}-\mathrm{O}$ \\
\hline $\begin{array}{l}\text { Исходные УНТ } \\
\text { Окисленные УНТ } \\
\left(140^{\circ} \mathrm{C}, 30 \text { ч }\right)\end{array}$ & 99,48 & 0,52 & - & 100 & - & - & - \\
$\begin{array}{l}\text { Предварительно очи- } \\
\text { щенные окисленные } \\
\text { УНТ }\left(140^{\circ} \mathrm{C}, 30 \text { ч) }\right.\end{array}$ & 99,41 & 0,59 & - & 98,20 & 1,50 & 0,30 & 0,00 \\
\hline
\end{tabular}

При разложении C1s-спектров определено, что на поверхности окисленных образцов кислород присутствует в виде гидроксильных групп, карбоксильные группы практически не идентифицируются. Следовательно, обработка в данной системе способствует присоединению относительно небольшого количества кислородсодержащих функциональных групп.

При этом согласно [15], первичной является реакция

$$
\mathrm{H}_{2} \mathrm{O}_{2}+\mathrm{H}_{2} \mathrm{O}_{2} \rightarrow \underset{\text { Интермедиаты }}{\left[\mathrm{OH}^{\bullet}, \mathrm{HO}_{2}^{\circ}\right]} \rightarrow \underset{2}{2} \mathrm{H}_{2} \mathrm{O}+\mathrm{O}_{2} .
$$

Образующиеся в этой реакции свободные радикалы являются окислителями в реакции, протекающей по схеме

$$
\mathrm{H}_{2} \mathrm{O}_{2}+\text { акцептор } \rightarrow \text { продукты реакции. }
$$

Разложение перекиси водорода на свободные радикалы в газовой фазе достаточно хорошо изучено и представлено совокупностью следующих реакций

$$
\mathrm{H}_{2} \mathrm{O}_{2}+\mathrm{M} \rightarrow 2 \mathrm{OH}^{\bullet}+\mathrm{M},
$$

где М - число всех молекул в реакционном объеме;

$$
\begin{gathered}
\mathrm{H}_{2} \mathrm{O}_{2}+2 \mathrm{OH}^{\bullet} \rightarrow \mathrm{HO}_{2}^{\bullet}+\mathrm{H}_{2} \mathrm{O} \\
\mathrm{HO}_{2}+\text { стенка } \rightarrow \mathrm{HO}_{2}^{\bullet} \text { адс } \\
\mathrm{OH}^{\bullet}+\text { стенка } \rightarrow \mathrm{OH}_{\text {адс } .}
\end{gathered}
$$




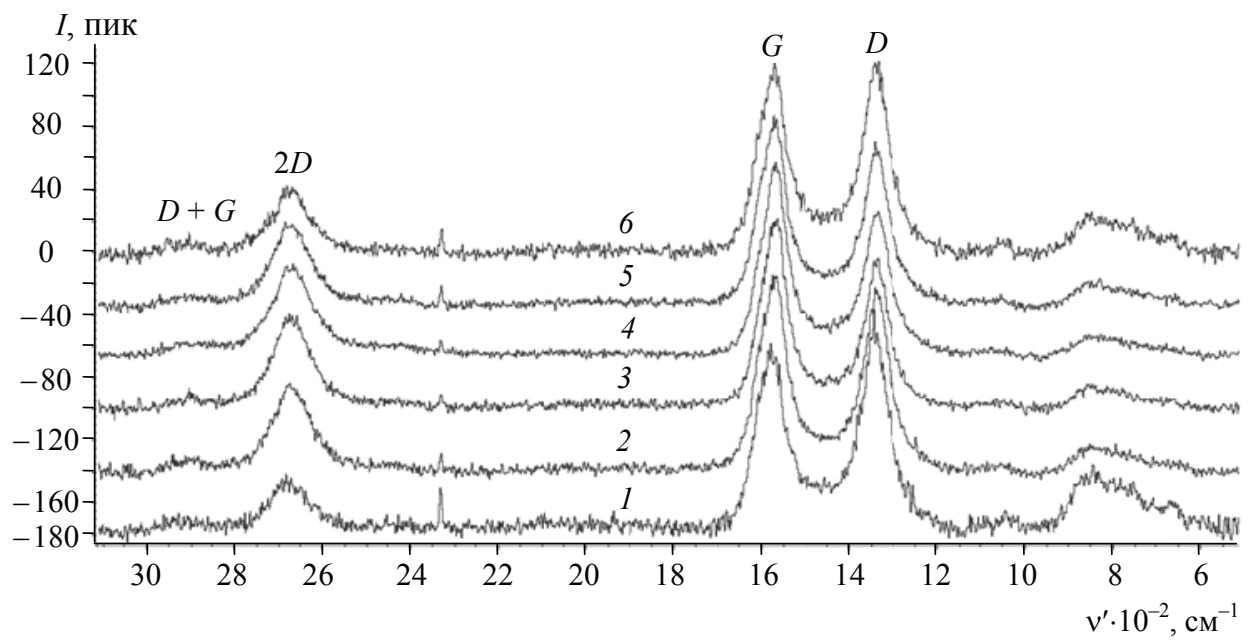

Рис. 4. Спектры КР исходных УНТ Таунит-М (1) и окисленных в парах перекиси водорода при $140{ }^{\circ} \mathrm{C}$ в течение 2 (2), 5 (3), 10 (4), 20 (5) и 30 ч (6) ( $v^{\prime}-$ сдвиг, КР)

Причем соотношение концентраций свободных радикалов в газовой фазе $\left[\mathrm{HO}_{2}{ }^{\circ}\right] /\left[\mathrm{OH}^{*}\right]$, согласно [15], составляет $10^{3} \ldots 10^{7}$. Преобладающие в системе свободные радикалы $\mathrm{HO}_{2}{ }^{\bullet}$ могут участвовать в формировании различных типов функциональных групп на поверхности УНТ.

Для выяснения влияния окисления в данной системе на целостность графеновых слоев УНТ исследованы спектры КР исходных и функционализированных материалов (рис. 4). Рассчитанные по ним соотношения $D / G$ представлены в табл. 2. При обработке в парах перекиси водорода как исходных, так и предварительно очищенных УНТ Таунит-М, в первые 10 ч окисления наблюдается снижение показателя дефектности графеновых слоев, затем он начинает расти, не достигая показателя, характерного для нефункционализированного материала даже через 30 ч.

Сопоставление данных ИК- и КР-спектров показывает, что алкильные группы, регистрируемые на ИК-спектрах (при 2933; 2854; 1452; $1378 \mathrm{~cm}^{-1}$ ), могут присутствовать как на поверхности углеродных нанотрубок, так на объемных включениях аморфной фазы. Снижение интенсивности соответствующих связям $\mathrm{C}-\mathrm{H}$ пиков связано не только с преобразованием алкильных групп в фенольные на поверхности УНТ, но и с удалением аморфного углерода. Предположения о закономерностях изменения степени дефектности УНТ в ходе окисления в парах перекиси водорода находят свое подтверждение в данных рентгеноструктурного анализа (рис. 5). Значение ширины пика на полувысоте $B_{0,5}$ снижается после пятичасового окисления УНТ, а затем вновь начинает возрастать.

Таблица 2

Оценка степени дефектности исходных и окисленных в парах перекиси водорода при $140{ }^{\circ} \mathrm{C}$ УНТ Таунит-М по данным спектров КР

\begin{tabular}{l|c|c|c|c|c|c}
\hline \multirow{2}{*}{ Вид УНТ } & \multicolumn{5}{|c}{ Значение соотношения $D / G$ на спектрах КР } \\
\cline { 3 - 6 } & 0 & 2 & 5 & 10 & 20 & 30 \\
\hline $\begin{array}{l}\text { Таунит-М } \\
\text { (исходные) }\end{array}$ & \multirow{2}{*}{1,190} & 0,930 & 0,781 & 0,728 & 0,854 & 0,938 \\
\cline { 1 - 6 } $\begin{array}{l}\text { Таунит-М } \\
\text { (очищенные) }\end{array}$ & & 0,938 & 0,801 & 0,786 & 0,896 & 0,959 \\
\hline
\end{tabular}




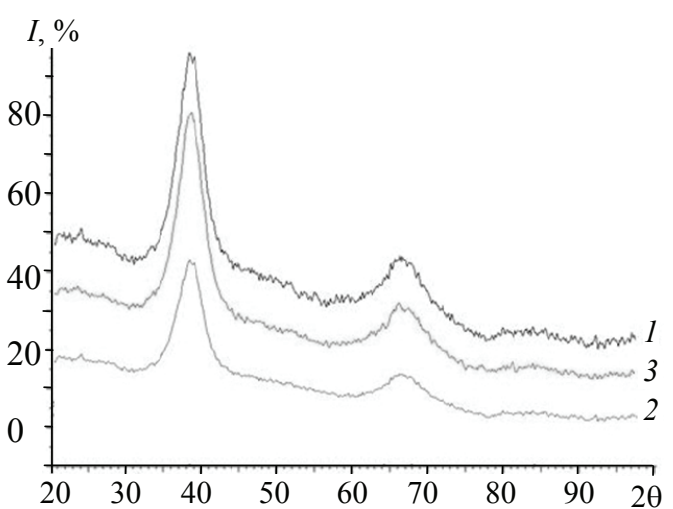

Рис. 5. Дифракторграммы исходных (1) и окисленных в парах перекиси водорода при $140{ }^{\circ} \mathrm{C}$ в течение 5 (2) и 20 ч (3) УНТ Таунит-М
По данным просвечивающей микроскопии видимых нарушений структуры боковых стенок УНТ не наблюдается даже после длительного окисления в парах перекиси водорода (рис. 6). Сохраняются и характерные геометрические параметры отдельных нанотрубок (длина и диаметр). На ПЭМ-изображениях обнаруживаются включения остатков металлоксидного катализатора в объеме некоторых УНТ. При более крупном увеличении заметны открытые концы нанотрубок.

По данным сорбтометрического анализа после обработки

в парах перекиси водорода величина удельной поверхности становится несколько ниже, чем для исходных УНТ. После обработки в течение 10 ч в данной системе

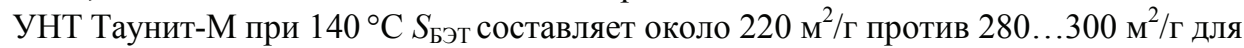
исходного материала. Это также хорошо согласуется с предположением об удалении поверхностной аморфной фазы. Характерной особенностью УНТ, функционализированных данным способом, является их структурирующее действие на некоторые полимерные матрицы [16]. По данным сканирующей электронной микроскопии (рис. 7) в матрице на основе «ПСК-1» окисленные в парах перекиси водорода УНТ находятся на поверхности ячеек из полисульфона размерами 0,5...1 мкм (см. рис. 7, a). Образцы полиуретана СКУ-8А, модифицированного функционализированными данным способом УНТ, обладают выраженной слоистой структурой (см. рис. 7, б).
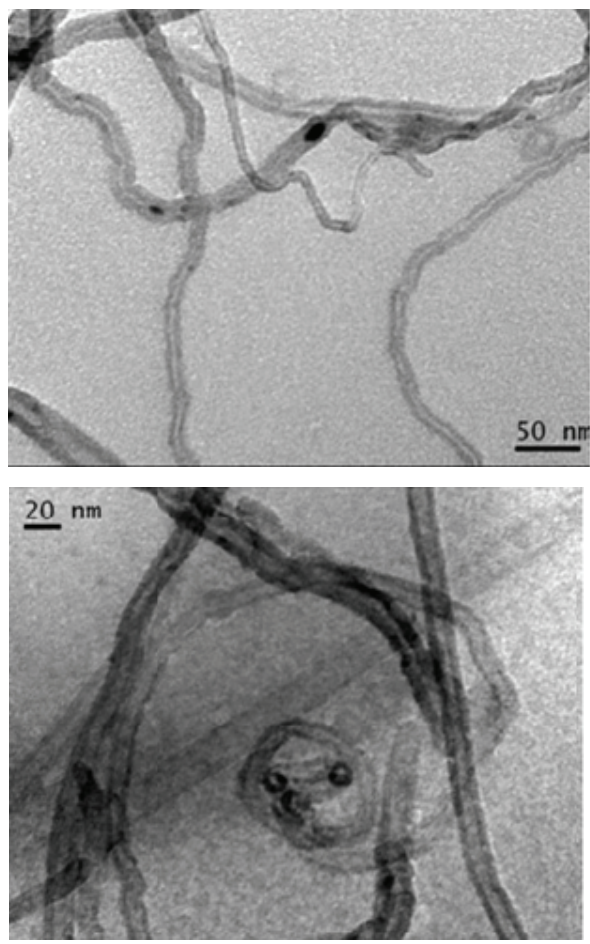

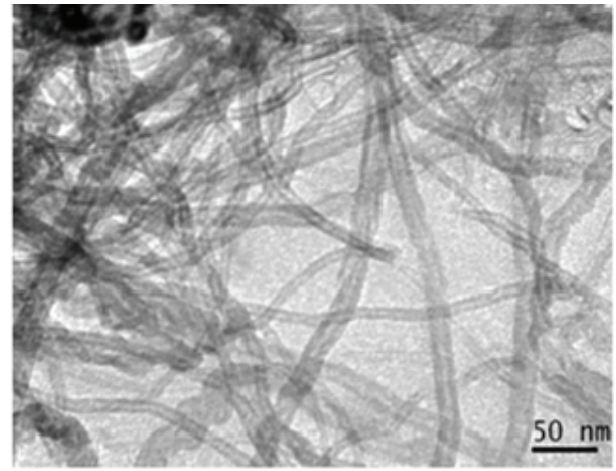

Рис. 6. ПЭМ-изображения УНТ Таунит-М после окисления в течение 30 ч в парах перекиси водорода при $140{ }^{\circ} \mathrm{C}$ 


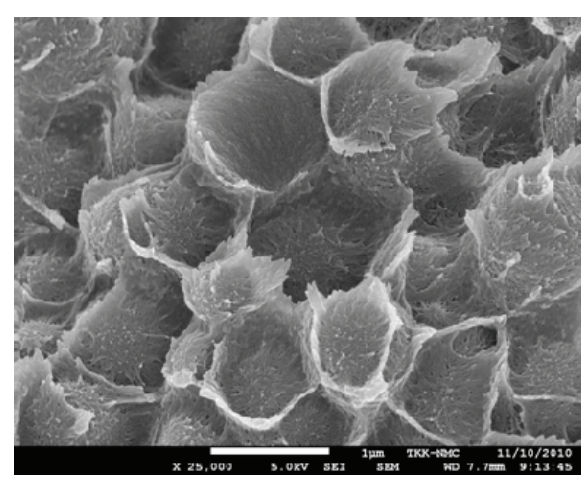

a)

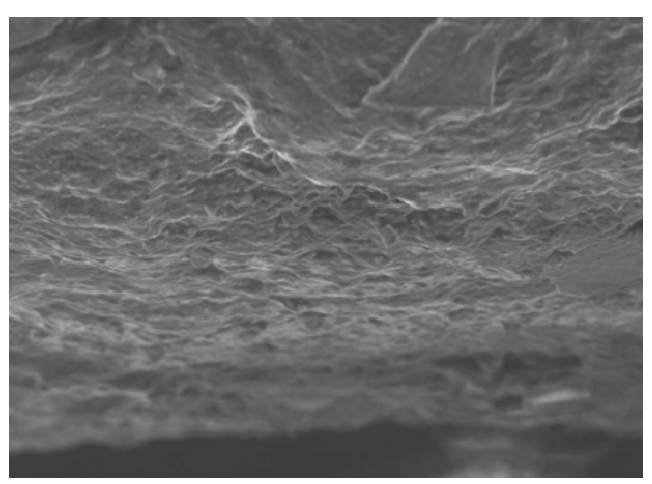

б)

Рис. 7. СЭМ-изображения нанокомпозитов смеси эпоксидной смолы и полисульфона (a) и полиуретана СКУ-8А (б) с функционализированными в парах перекиси водорода УНТ Таунит-М (увеличение: $a-$ в $50000 ; \sigma-$ в 1500 раз)

Из-за невысокой степени функционализации сильное взаимодействие УНТ с молекулами полимерной матрицы невозможно, поэтому во всех исследованных случаях не удается достичь равномерного распределения. Однако диспергирование УНТ не всегда является необходимым условием проявления наилучших эффектов с позиций влияния на свойства композитов. В данном случае, структурирование полимерной матрицы наряду с образованием в ней сетки из агломератов УНТ приводит к улучшению радиоэкранирующих и электропроводящих свойств модифицируемых материалов, расчет величины ослабления проведен для образца на основе синтетического каучука (СК) толщиной 2 мм (табл. 3).

Уровень ослабления, который характерен для композитов на основе окисленных в парах перекиси водорода УНТ с концентрацией 3 \% масс., обычно достигается при концентрациях УНТ порядка $9 . .15 \%$ масс. [17, 18].

Таким образом, для применения в составе композитов, обладающих электропроводящими, радиопоглощающими и/или радиоэкранирующими свойствами, УНТ целесообразно подвергать окислению в парах перекиси водорода.

Данный способ функционализации экологически безопасен, поскольку при его реализации не образуется требующих обезвреживания и утилизации отходов. Дополнительным преимуществом используемой окислительной системы является ее умеренная коррозионная активность. Ввиду простоты процесса и востребованности его целевого продукта целесообразными являются масштабирование и реализация в промышленных масштабах.

Таблица 3

Значения величин действительной $\varepsilon^{\prime}$ и мнимой $\varepsilon^{\prime \prime}$ частей диэлектрической проницаемости, тангенса диэлектрических потерь $\delta$ композитов, содержащих 3 \% масс. УНТ, при частоте 10 ГГц

\begin{tabular}{l|c|c|c|c|c}
\hline \multicolumn{1}{c|}{ Образец } & $\varepsilon^{\prime}$ & $\varepsilon^{\prime \prime}$ & $\operatorname{tg} \delta$ & $\begin{array}{c}\text { Коэффициент } \\
\text { ослабления, } \\
\text { дБ }\end{array}$ & $\begin{array}{c}\text { Поверхностное } \\
\text { сопротивление } \\
\text { кОм/кВ }\end{array}$ \\
\hline $\mathrm{CK} /$ исходные УНТ & 13,80 & 18,59 & 1,34 & 12,93 & 1,2 \\
$\begin{array}{l}\text { СК/УНТ, функционали- } \\
\text { зированные в парах } \mathrm{H}_{2} \mathrm{O}_{2}\end{array}$ & 41,38 & 44,56 & 1,07 & 18,68 & 0,7 \\
\hline
\end{tabular}




\section{Заключение}

Обработка многослойных углеродных нанотрубок в парах перекиси водорода при темпратуре $140{ }^{\circ} \mathrm{C}$ способствует формированию минимального количества кислородсодержащих, преимущественно гидроксильных групп, что подтверждается методами ИК- и РФЭ-спектроскопии.

Функционализированные данным способом УНТ обладают упорядоченными графеновыми слоями. Минимальная степень дефектности, по данным спектроскопии КР, достигается при выдержке в течение 10 ч УНТ в парах перекиси водорода при указанной температуре.

Окисленные в парах перекиси водорода УНТ образуют упорядоченно расположенные агломераты, что способствует особому типу структурирования полимерных матриц на основе полисульфона и синтетического каучука. Результатом данного эффекта являются выраженные радиэкранирующие и электропроводящие свойства нанокомпозитов.

Автор выражает благодарность сотрудникам Центр коллективного пользования научным оборудованием МГУ им М. В. Ломоносова, ФГУП «ВИАМ», НИТУ «МИСИС», УИЦ «Нанотехнологии и наноматериаль» ТГУ им. Г. Р. Державина за помощь в диагностике свойств образиов УНТ и композитов на их основе.

Работа выполнена в рамках государственной поддержки развития кооперации российских высших учебных заведений, государственных научных учреждений и организаций, реализующих комплексные проекты по созданию высокотехнологичного производства, осуществляемой в соответствии c постановлением Правительства РФ от 9.04.2010 2 2. № 218 (договор № 02.G25.31.0123 om 14.08.2014 2.).

\section{Список литературы}

1. Multiscale Fiber-Reinforced Thermoplastic Composites Incorporating Carbon Nanotubes: A Review / A. M. Diez-Pascual [et al.] // Current Opinion in Solid State and Materials Science. - 2014. - Vol. 18, No. 2. - P. 62 - 80.

2. Rajeshwari, P. Microstructure and Mechanical Properties of Multiwall Carbon Nanotubes Reinforced Polymer Composites / P. Rajeshwari // Materials Today: Proceedings. - 2015. - Vol. 2, No. 4-5. - P. $3598-3604$.

3. Ailan, A. R. Multiscale Modeling of the Effect of Waviness and Agglomeration of Cnts on the Elastic Properties of Nanocomposites / A. R. Ailan, S. El-Borgi, S. A. Meguid // Computational materials Science. - 2016. - Vol. 117. - P. 195 - 204.

4. Polymer Nanocomposites Based on Functionalized Carbon Nanotubes / N. G. Sahoo [et al.] // Progress in Polymer Science. - 2010. - Vol. 35. - P. 837 - 867.

5. Suri, A. The Superiority of Air Oxidation Over Liquid-Phase Oxidative Treatment in the Purification of Carbon Nanotubes / A. Suri, K. S. Coleman // Carbon. 2011. - Vol. 49. - P. 3031 - 3038.

6. A Highly Efficient Gas-Phase Route for the Oxygen Functionalization of Carbon Nanotubes Based on Nitric Acid Vapor / W. Xia [et al.] // Carbon. - 2009. Vol. 47. - P. $919-922$.

7. Fracture Mechanisms of Epoxy Filled with Ozone Functionalized Multi-Wall Carbon Nanotubes / L.-C. Tang [et al.] // Composites Science and Technology. - 2011. Vol. 72. - P. $7-13$.

8. Nagiev, T. M. Coherent Synchronized Oxidation Reactions by Hydrogen Peroxide / T. M. Nagiev. - Amsterdam; Boston : Elsevier, 2006. - 325 p.

9. A Kinetic Study of Vapor-Phase Cyclohexene Epoxidation by $\mathrm{H}_{2} \mathrm{O}_{2}$ over Mesoporous TS-1 / S. Kwon [et al.] // Journal of Catalysis. - 2015. - Vol. 326. P. $107-115$. 
10. Graphite and Carbon Black Materials as Catalysts for Wet Peroxide Oxidation / C. M. Domínguez [et al.] // Applied Catalysis B: Environmental. - 2014. - Vol. 144. P. $599-606$.

11. Carbon Nanotubes as Catalysts for Catalytic Wet Peroxide Oxidation of Highly Concentrated Phenol Solutions: Towards Process Intensification / M. T. Pinho [et al.] // Applied Catalysis B: Environmental. - 2015. - Vol. 165. - P. 706 - 714.

12. Some Aspects of Carbon Nanotubes Technology / A. V. Melezhyk [et al.] // Nanosystems: Physics, Chemistry, Mathematics. - 2013. - Vol. 4, No. 2. - P. 247 - 259.

13. Горский, С. Ю. Газофазная функционализация углеродных нанотрубок: проблемы реализации метода / С. Ю. Горский, Т. П. Дьячкова, Е. А. Буракова // Науч.-техн. ведомости С.-Петербург. гос. политехн. университета. - 2014. Вып. 1(190). - С. 108 - 112.

14. Михеева, Е. В. Физическая и коллоидная химия / Е. В. Михеева, Н. П. Пикула. - Томск : Изд-во ТПУ, 2009. - 267 с.

15. Нагиев, Т. М. Сопряженные реакции окисления перекисью водорода / Т. М. Нагиев // Успехи химии. - 1985. - Вып. 10. - С. 1654 - 1673.

16. Каблов, Е. Н. Перспективы использования углеродсодержащих наночастиц в связующих для полимерных конструкционных материалов / Е. Н. Каблов, С. В. Кондрашов, Г. Ю. Юрков // Российские нанотехнологии. - 2013. - Т. 8, № 3-4. - C. $24-42$.

17. The Influence of Single-Walled Carbon Nanotube Structure on the Electromagnetic Interference Shielding Efficiency of its Epoxy Composites / Y. Huang [et al.] // Carbon. - 2007. - Vol. 45, No. 8. - P. 1614 - 1621.

18. Microwave Attenuation of Multiwalled Carbon Nanotube-Fused Silica Composites / X. Changshu [et al.] // Applied physics letters. - 2005. - Vol. 87. P. 123103.

\section{Oxidation of Multiwalled Carbon Nanotubes by Hydrogene Peroxide Vapor: Laws and Effects}

\section{T. P. Dyachkova ${ }^{1}$, Yu. A. Khan ${ }^{1}$, N. V. Orlova ${ }^{2}$, S. V. Kondrashov ${ }^{3}$}

Departments: "Technology and Methods of Nanoproducts Manufacturing" (1); Technological Processes, Devices and Technosphere Safety" (2); TSTU; All Russian Scientific Research Institute of Aviation Materials, Moscow (3); dyachkova_tp@mail.ru

Keywords: carbon nanotubes; composite materials; defect rate; functionalization; gas-phaseprocess; oxidation.

Abstract: The regularities of multiwalled carbon nanotubes (CNTs) oxidation by hydrogen peroxide vapor were researched. The raw and the treated CNTs were studied by electron microscopy, IR-, XPS- and Raman spectroscopy, XRD, TG and specific surface area analysis. The effect of temperature conditions, the duration of the process and the metal oxide catalyst particles on the qualitative and quantitative content of the surface functional groups formed during the oxidation of CNTs by hydrogen peroxide vapor was shown. The grapheme layer structure changing was studied. It was shown that polymer composites based on functionalized CNTs have improved radio shielding and electrical conductivity. The proposed method for CNTs treating is cost-effective, environmentally friendly and can be easily scaled. 


\section{References}

1. Diez-Pascual A.M., Naffakh M., Marco C., Gomez-Fatou M.A., Ellis G.J. Multiscale fiber-reinforced thermoplastic composites incorporating carbon nanotubes: A review, Current Opinion in Solid State and Materials Science, 2014, vol. 18, no. 2, pp. 62-80.

2. Rajeshwari P. Microstructure and Mechanical Properties of Multiwall Carbon Nanotubes Reinforced Polymer Composites, Materials Today: Proceedings, 2015, vol. 2, no. 4-5, pp. 3598-3604.

3. Ailan A.R., El-Borgi S., Meguid S.A. Multiscale modeling of the effect of waviness and agglomeration of CNTs on the elastic properties of nanocomposites, Computational materials Science, 2016, vol. 117, pp. 195-204.

4. Sahoo N.G., Rana S., Cho J.W., Li L., Chan S.H. Polymer nanocomposites based on functionalized carbon nanotubes, Progress in Polymer Science, 2010, vol. 35, pp. 837-867.

5. Suri, A., Coleman K.S. The superiority of air oxidation over liquid-phase oxidative treatment in the purification of carbon nanotubes, Carbon, 2011, vol. 49, pp. 3031-3038.

6. Xia W., Jin C., Kundu S., Muhler M. A highly efficient gas-phase route for the oxygen functionalization of carbon nanotubes based on nitric acid vapor, Carbon, 2009. vol. 47, pp. 919-922.

7. Tang L.-C., Zhang H., Han J.-H., Wu X.-P., Zhang Z. Fracture mechanisms of epoxy filled with ozone functionalized multi-wall carbon nanotubes, Composites Science and Technology, 2011, vol. 72, pp. 7-13.

8. Nagiev T.M. Coherent Synchronized Oxidation Reactions by Hydrogen Peroxide, Amsterdam; Boston: Elsevier, 2006, 325 p.

9. Kwon S., Schweitzer N.M., Park S., Stair P.C., Snurr R.Q. A kinetic study of vapor-phase cyclohexene epoxidation by $\mathrm{H}_{2} \mathrm{O}_{2}$ over mesoporous TS-1, Journal of Catalysis, 2015, vol. 326, pp. 107-115.

10. Domínguez C.M., Ocón P., Quintanilla A., Casas J.A., Rodríguez J.J. Graphite and carbon black materials as catalysts for wet peroxide oxidation, Applied Catalysis B: Environmental, 2014, vol. 144, pp. 599-606.

11. Pinho M.T., Gomes H.T., Ribeiro R.S., Faria J.L., Silva A.M. Carbon nanotubes as catalysts for catalytic wet peroxide oxidation of highly concentrated phenol solutions: towards process intensification, Applied Catalysis B: Environmental, 2015, vol. 165, pp. 706-714.

12. Melezhyk A.V., Rukhov A.V., Tugolukov E.N., Tkachev A.G. Some aspects of carbon nanotubes technology, Nanosystems: Physics, Chemistry, Mathematics, 2013. vol. 4, no. 2, pp. 247-259.

13. Gorskii S.Yu., D'yachkova T.P., Burakova E.A. [Gas phase functionalization of carbon nanotubes: problems of implementation of the method], Nauchnotekhnicheskie vedomosti Sankt-Peterburgskogo gosudarstvennogo politekhnicheskogo universiteta [Scientific and technical sheets of St. Petersburg State Polytechnic University], 2014, ussue 1(190), pp. 108-112. (In Russ.)

14. Mikheeva E.V., Pikula N.P. Fizicheskaya i kolloidnaya khimiya [Physical and Colloid Chemistry], Tomsk: Izd-vo TPU, 2009, 267 p. (In Russ.)

15. Nagiev T.M. [Conjugate reaction of hydrogen peroxide oxidation], Uspekhi khimii [Russian Chemical Reviews], 1985, ussue 10, pp. 1654-1673. (In Russ.)

16. Kablov E.N., Kondrashov S.V., Yurkov G.Yu. [Prospects of using carbonaceous nanoparticles in binders for polymer composites], Rossiiskie nanotekhnologii [Nanotechnologies in Russia], 2013, vol. 8, no. 3-4, pp. 24-42. (In Russ., abstract in Eng.)

17. Huang Y., Ning L., Yanfeng M., Feng D., Feifei L., Xiaobo H., Xiao L., Hongjun G., Yongsheng C. The influence of single-walled carbon nanotube structure on 
the electromagnetic interference shielding efficiency of its epoxy composites, Carbon, 2007, vol. 45, no. 8, pp. 1614-1621.

18. Changshu X., Yubai P., Xuejian L., Xingwei S., Xiaomei S., Jingkun G. Microwave attenuation of multiwalled carbon nanotube-fused silica composites, Applied physics letters, 2005, vol. 87, pp. 123103.

\section{Oxydierung der vielschichtigen Kohlenstoffnanoröhre in den Paaren des Wasserstoffperoxides: Gesetzmäßigkeiten und Effekte}

Zusammenfassung: Es sind die Gesetzmäßigkeiten der Oxydierung der vielschichtigen Kohlenstoffnanoröhre in den Paaren des Wasserstoffperoxides erlernt. Die originalen und bearbeiteten Kohlenstoffnanoröhre sind von den Methoden der elektronischen Mikroskopie, IR- und RPhE-Spektroskopie, der Spektroskopie des kombinatorischen Zerstreuens, der Röntgenstrukturanalyse, Thermoschweremessung untersucht. Es ist der Einfluss der Temperaturbedingungen, der Dauer des Prozesses und des Vorhandenseins der Beimischungen des Metalloxidkatalysators auf die qualitativen und quantitativen Zusammensetzung der oberflächlichen funktionalen Gruppen, die sich bei der Oxydierung der Kohlenstoffnanoröhre in den Paaren des Wasserstoffperoxides entwickeln, bestimmt. Es ist die Einschätzung des Einflusses der gegebenen Weise der Oxydierung auf die Struktur der Graphemschichten der Kohlenstoffnanoröhre durchgeführt. Es ist gezeigt, dass die polymeren Verbundwerkstoffe auf Grund der funltionalisierten Kohlenstoffnanoröhre die verbesserten Radioschutz- und Elektroleiteigenschaften haben. Die vorschlagende Weise der Bearbeitung der Kohlenstoffnanoröhre ist ökonomisch vorteilhaft, ökologisch rein und kann leicht ausgemessen sein.

\section{Oxydation des nanotubes carboniques multicouches dans les vapeurs de peroxyde d'hydrogène: régularités et effets}

Résumé: Sont étudiées les régularités de l'oxydation des nanotubes carboniques multicouches dans les vapeurs de peroxyde d'hydrogène. Les nanotubes carboniques origines et usinées sont étudiées par les méthodes de la microscopie électronique, de IR et de RPE spectroscopie, de spectroscopie raman, de l'analyse de la structure roentgen, etc. Est définie l'nfluence des conditions de température, de la durée du processus et de la présence d'impuretés du catalyseur sur la composition qualitative et quantitative des groupes fonctionnels de surface, formés lors de l'oxydation de nanotubes. Est effectuée l'évaluation de l'impact de cette méthode d'oxydation sur la structure des couches de graphène des nanotubes carboniques. La méthode proposée pour le traitement des nanotubes carboniques est rentable du point de vue de l'économie.

Авторы: Дьячкова Татьяна Петровна - кандидат химических наук, доцент кафедры «Техника и технологии производства нанопродуктов»; Хан Юлиан Александрович - студент; Орлова Наталия Вячеславовна - кандидат технических наук, доцент кафедры «Технологические процессы, аппараты и техносферная безопасность», ФГБОУ ВО «ТГТУ»; Кондрашов Станислав Владимирович кандидат физико-математических наук, ведущий научный сотрудник ФГУП «Всероссийский научно-исследовательский институт авиационных материалов», г. Москва.

Рецензент: Рухов Артем Викторович - доктор технических наук, исполняющий обязанности заведующего кафедрой «Химия и химические технологии», ФГБОУ ВО «ТГТУ». 